\title{
Perovskite light-emitting/detecting bifunctional fibres for wearable LiFi communication
}

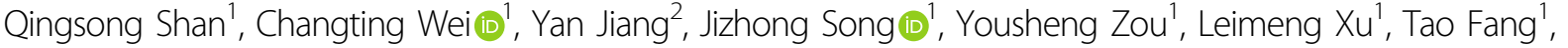

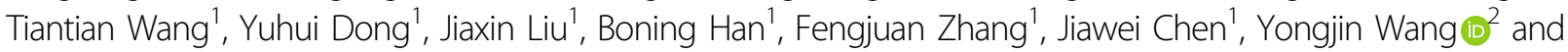 \\ Haibo Zeng (10
}

\begin{abstract}
Light fidelity (LiFi), which is emerging as a compelling technology paradigm shifting the common means of highcapacity wireless communication technologies, requires wearable and full-duplex compact design because of its great significance in smart wearables as well as the 'Internet of Things'. However, the construction of the key component of wearable full-duplex LiFi, light-emitting/detecting bifunctional fibres, is still challenging because of the conflicting process between carrier separation and recombination, as well as the highly dynamic film-forming process. Here, we demonstrate light-emitting/detecting bifunctional fibres enabled by perovskite QDs with hybrid components. The hybrid perovskite inks endow fibres with super-smooth QD films. This, combined with the small exciton binding energy and high carrier mobility of perovskite QDs, enables successful integration of electroluminescence and photodetection into monofilaments. The bifunctional fibres possess the narrowest electroluminescence full width at half maximum of $\sim 19 \mathrm{~nm}$ and, more importantly, the capability for simultaneously transmitting and receiving information. The successful fabrication of narrow emission full-duplex LiFi fibres paves the way for the fabrication and integration of low crosstalk interoperable smart wearables.
\end{abstract}

\section{Introduction}

A vast amount of data is generated every day in this information era, which has triggered the development of new high-speed wireless data communication technologies, such as the fifth generation of cellular systems $(5 \mathrm{G})$ and light fidelity (LiFi). This, combined with the emerging fields of the 'Internet of Things' (IoT) and big data, persistently pushes the miniaturization and densification of wireless communication terminals. In this context, the targeted design of wearable LiFi with the merits of portability, conformability, and safe and high-speed wireless

\footnotetext{
Correspondence: Jizhong Song (songjizhong@njust.edu.cn) or Yongjin Wang (wangyj@njupt.edu.cn) or Haibo Zeng (zeng.haibo@njust.edu.cn) ${ }^{1}$ MIIT Key Laboratory of Advanced Display Materials and Devices, Institute of Optoelectronics \& Nanomaterials, School of Materials Science and Engineering, Nanjing University of Science and Technology, Nanjing 210094, China ${ }^{2}$ Peter Grünberg Research Center, Laboratory of Broadband Wireless Communication and Sensor Network Technology, Ministry of Education, Nanjing University of Posts and Telecommunications, Nanjing 210003, China These authors contributed equally: Qingsong Shan, Changting Wei, Yan Jiang
}

communication will have boundless prospects in IoT terminals. Nevertheless, a typical LiFi system always consists of considerable quantities of transmitters (LEDs) and receivers (PDs) to meet the demand for high-capacity wireless communication and multiuser interaction. This definitely increases the complexity and volume of LiFi equipment, hindering the construction of wearable LiFi. The construction of full-duplex (bilateral real-time communication capable) wearable LiFi will be helpful for obtaining higher data rates and more portable IoT terminals ${ }^{1}$.

Currently, rapid advances in material science and processing technology have made versatile wearables with functions such as health care, data transfer, energy storage, and military and fashion applications possible ${ }^{2-9}$. Therein, fibres have been proven to be excellent platforms for the construction of versatile functional devices ${ }^{9-17}$ as well as carriers of receiver and emitter diodes in wearable optical communication systems ${ }^{4}$. In terms of the data 
transmission part in wearable $\mathrm{LiFi}$, electroluminescent (EL) fibres based on inorganic phosphors ${ }^{18}$, organic materials $^{15,19}$, and polymers ${ }^{9,20}$ have already been reported, but they all have some shortcomings and are not suitable for the construction of wearable LiFi. For example, EL fibres based on inorganic phosphors require an alternating current (AC) field to create polarization currents to realise electroluminescence, and they suffer from inhomogeneous emission and a high operating voltage, which prevent them from being safely and conveniently integrated into textiles. Polymer light-emitting electrochemical cells usually have a slow turn-on process because of the $\mathrm{p}-\mathrm{i}-\mathrm{n}$ junction formation requiring time for the diffusion of mobile ions, which is not suitable for data transmission. The widely used thermal evaporation process in the fabrication of OLED fibres is costly. More importantly, a broad EL full width at half maximum (FWHM) is ubiquitous in the above devices, and the overlap of EL spectra between different EL devices will certainly bring about channel crosstalk in the operation of the LiFi system based on wavelength-division multiplexing technology.

Apart from the demands for high luminescence quality and an inexpensive fabrication process, integrating the detection function into a single EL fibre is also necessary for wearable $\mathrm{LiFi}$. Although light-emitting/detecting bifunctional devices have been realised on rigid substrates, including in double-heterojunction $\mathrm{CdS} / \mathrm{CdSe} / \mathrm{ZnSe}$ nanorod $^{21}$, bilayer $\mathrm{MoTe}_{2} \mathrm{p}-\mathrm{n}$ junction ${ }^{22}$, and InGaN/ $\mathrm{Al}_{0.10} \mathrm{Ga}_{0.90} \mathrm{~N}$ multiple-quantum-well ${ }^{23}$ based devices, these device structures and materials are not suitable for wearable light-emitting/detecting bifunctional fibres. The construction of such bifunctional devices is still challenging because of the conflicting process between carrier separation and recombination as well as the highly dynamic film-forming process ${ }^{24}$; suitable materials and device structure are needed. Quantum dots (QDs), particularly metal halide perovskite QDs, have been extensively used for the construction of optoelectronic devices due to their excellent photoelectric properties ${ }^{25-30}$. Considering the easily photogenerated charges of perovskite QDs and their easily adjustable ink components ${ }^{31}$, the challenge of the conflicting process between carrier separation and recombination, as well as the highly dynamic film-forming process for constructing lightemitting/detecting bifunctional fibres, can be handled.

Here, we demonstrate a perovskite QD-based lightemitting/detecting bifunctional fibre obtained through a hybrid strategy and a facile, reproduceable solution assembly process. The hybrid perovskite inks improved the film-forming process, resulting in super-smooth QD films as well as successful fabrication of electroluminescent fibres. The green-emitting perovskite fibre has an FWHM of $\sim 19 \mathrm{~nm}$, which is the narrowest electroluminescence linewidth among all electroluminescent fibres. The emitting perovskite fibre can simultaneously receive information due to the small exciton binding energy and high carrier mobility of the perovskite QDs. The successful fabrication of narrow emission full-duplex LiFi fibres paves the way for the fabrication and integration of low crosstalk interoperable smart wearables.

\section{Results \\ Fabrication of perovskite fibres}

The coaxial quantum dot light-emitting fibre (QLEF) was fabricated layer by layer through a typical dip-coating process. As shown in Fig. 1a, a transparent PET fibre was used as the substrate for the construction of the coaxial structure flexible QLEF. The transparent electrodes and QD layer were subsequently dip-coated on the $0.3 \mu \mathrm{m}$ diameter polyethylene terephthalate (PET) fibre. It is well known that both appropriate viscosity and surface tension of the solution are crucial for the construction of a highquality electroluminescent (EL) layer in the dip-coating process. Generally, it is difficult to dip-coat homogeneous and smooth perovskite QD films via the regulation of the solvent and concentration. It is difficult to control the QD film-forming stage in the dip-coating process because the surface tension of QD ink is rather low, which usually makes QDs spread easily ${ }^{24}$. For a single-step dip-coating process, QDs typically form a network structure decorated by isolated islands of aggregated QDs (Supplementary Fig. S1). For a multiple-step dip-coating process, the QD film usually has a large surface roughness. The heterogeneous structure will create a short-circuit current and joule heating under an electric field when applied as a luminous layer of EL devices, which will seriously deteriorate the luminance uniformity of EL fibres or even disable the EL fibre device. Herein, we propose a hybrid QD ink system, which consists of poly[bis(4-phenyl) (2,5,6-trimethylphenyl)amine poly(triarylamine) (PTAA), 1,3,5-tri[(3-pyridyl)-phen-3-yl]benzene (TmPyPB), and perovskite QDs (Fig. 1b). The green emission of pure QD ink changes to cyan after the addition of PTAA and TmPyPB under UV irradiation. Meanwhile, the emission peak of the perovskite QDs is slightly blue shifted (Supplementary Fig. S2), which is probably because of the change in the average size of the self-assembled QDs in the organic matrix ${ }^{32}$.

Figure 1c demonstrates AFM images of dip-coated films obtained by using the pure QD and hybrid QD inks under the same procedure. The as-prepared hybrid QD film exhibits a super-smooth surface with a roughness of $1.9 \mathrm{~nm}$, while the film dip-coated with pure QDs has a rather rough surface with a roughness greater than $10 \mathrm{~nm}$. This is further confirmed by the PL mapping results (Supplementary Fig. S3). The fused NCs in the hybrid film 
a

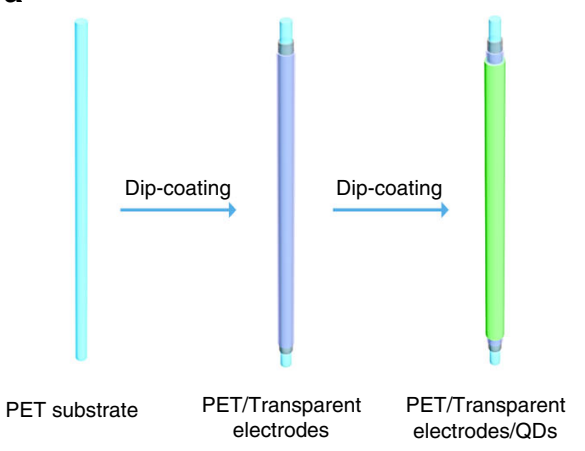

C

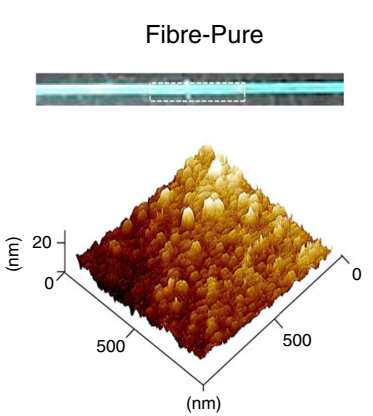

e

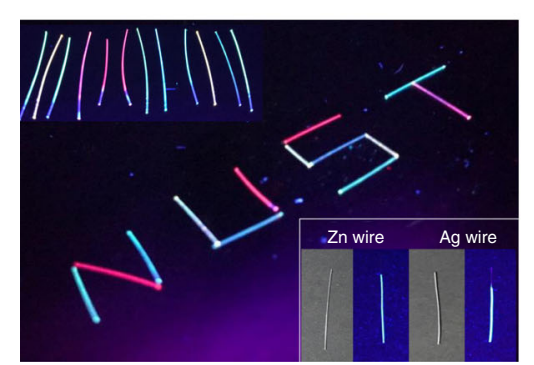

b

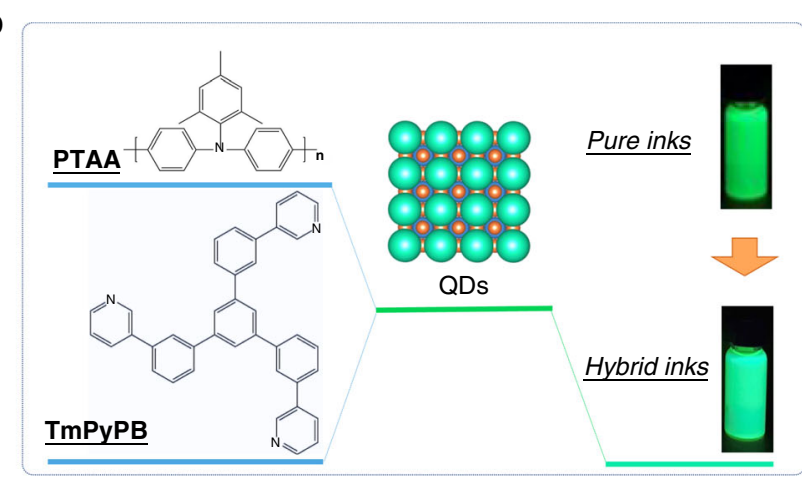

d

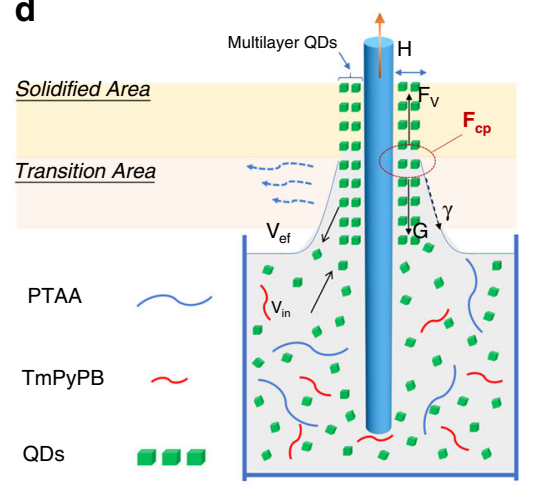

g
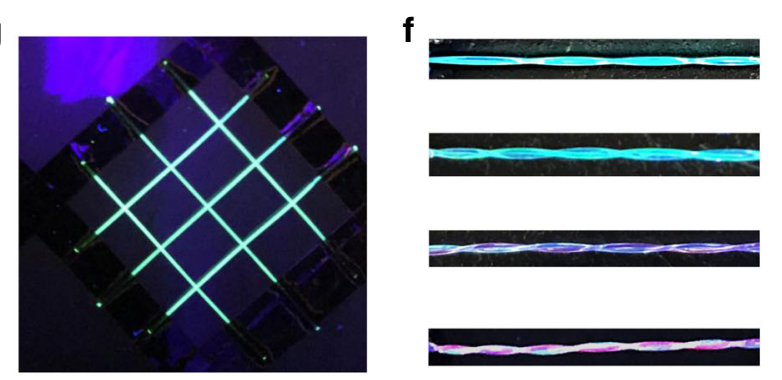

Fig. 1 Fabrication of LED-photodetector bifunctional multilayer perovskite fibres. a Schematic illustration of the multiple dip-coating process of transparent electrodes and QDs in the fabrication of a perovskite fibre. $\mathbf{b}$ Schematic of the hybrid strategy of QD inks. c Photographs of asprepared QD fibres, and corresponding AFM images. d Schematic illustration of the dynamic dip-coating process. e Perovskite fibres arranged into a 'NUST' pattern under UV illumination (RGB emission fibres were made with hybrid $\mathrm{CsPb}_{3}, \mathrm{CsPbBr}_{3}$, and $\mathrm{CsPb}(\mathrm{Br} / \mathrm{Cl})_{3}$ inks). Insert, perovskite fibres being prepared on $\mathrm{Zn}$ and $\mathrm{Ag}$ wires, and their corresponding PL images under UV illumination. $\mathbf{f}$ Photographs of the perovskite fibres woven together into a lattice under UV illumination. $\mathbf{g}$ Photograph of perovskite fibres assembled into one multicolour emission fibre under UV illumination.

and the possible coupling between QDs may also help improve the luminescent properties with the assistance of multiexciton resonances ${ }^{33,34}$. We believe that the successful fabrication of this super-smooth film is probably due to the improved film-forming process of the hybrid QD ink. Figure 1d illustrates a typical dip-coating process. In brief, the fibre is first dipped into and wetted by the QD ink, followed by the vertical withdrawal process. In the film-forming process in dip-coating, the film thickness is controlled by the coefficient of the gravity force $(G)$, viscous drag $\left(\mathrm{F}_{\mathrm{v}}\right)$ and liquid-vapour surface tension $(\gamma)^{24,35}$. Typically, the dip-coating process can be divided into the transition stage and solidified stage. Draining and evaporation of the solvent accompany the balance between the QD efflux and influx below the liquid-gas-solid three-phase contact line in the transition stage. Usually, solvent evaporation often results in many pores in the compliant film. The pore-containing structure collapses in the solidified stage due to the effect of the capillary force $\left(\mathrm{F}_{\mathrm{cp}}\right)$, which is proportional to the surface tension and viscosity of the ink. Inevitably, the viscosity and surface tension of hybrid QD inks are increased after the addition of organic components (Supplementary Fig. S4 and Table 1), which creates a greater $\mathrm{F}_{\mathrm{cp}}$, thereby forming 
Table 1 Comparison of the FWHM of reported fibreshaped electroluminescent devices.

\begin{tabular}{lllll}
\hline $\begin{array}{llll}\text { Device Type } \\
\text { matting } \\
\text { materials }\end{array}$ & FWHM (nm) & $\begin{array}{l}\text { Peak } \\
\text { position }(\mathbf{n m})\end{array}$ & Cation \\
\hline OLED & Alq $_{3}$ /super yellow & $113 / 67$ & $518 / 531$ & 36 \\
OLED & Super Yellow & 93 & 545 & 15 \\
OLED & Alq & $\sim 86$ & 536 & 19 \\
OLED & Super Yellow & $\sim 86$ & 550 & 37 \\
PLEC & PF-B/ETT-15/LiTf & $\sim 100$ & $496-550$ & 9 \\
ACPEL & ZnS:Cu, Cl & 85 & 493 & 18 \\
Our work & Perovskite QDs & $\sim 19$ & $\sim 516$ & - \\
\hline
\end{tabular}

a super-smooth film through a cooperative effect of the gravity force, viscous drag and liquid-vapour surface tension. In addition, the gap-filling effect of small molecules and polymers in QD inks also contributes to a super-smooth film ${ }^{38}$. In contrast, the rough film dipcoated using pure QD inks was probably caused by aggregation of QDs, thermal expansion and solvent evaporation during the multiple dip-coating and thermal treatment processes. As a result, dip-coated fibres with homogeneous photoluminescence can be obtained. As shown in Fig. 1e, the perovskite fibres can be arranged into an 'NUST' pattern and exhibit homogeneous PL properties. In addition, such a composite strategy can be extended to various kinds of substrates, such as $\mathrm{Ag}$ wire and $\mathrm{Zn}$ wire. The bright and uniform photoluminescence under UV light is shown in the insert of Fig. 1e. The photoluminescent perovskite fibres can also be assembled into luminous textiles (Fig. 1f) as well as one fibre to achieve multicolour woven structures (Fig. 1g), showing great potential for the fabrication of perovskite EL fibres.

\section{Discussion}

\section{Electroluminescence properties of the perovskite fibres}

As shown in Fig. 2a, the device structure of EL fibres was designed according to the typical planar device structure. PTAA, TPBi, and $\mathrm{Liq} / \mathrm{Al}$ were selected as the hole transport layer (HTL), electron transport layer (ETL), and cathode, respectively. PEDOT:PSS (Clevios PH1000) was used as a high conductivity transparent anode. As shown in Fig. 2a, the structure of the perovskite EL fibre was further characterized by a FIB-SEM system. The asprepared perovskite fibre consists of $\sim 150 \mathrm{~nm}$ PEDOT: PSS and PTAA as the HTL, a $65 \mathrm{~nm}$ thick QD layer (estimated by the thickness of the hybrid QD film) as the emitting layer, $50 \mathrm{~nm}$ TPBi as the ETL, and $3 \mathrm{~nm} \mathrm{Liq} \mathrm{and}$ $100 \mathrm{~nm} \mathrm{Al}$ as the cathode. For a fibre-shaped device, the sheet resistance of the anode seriously affects the luminance uniformity. Herein, a multi-dip-coating process is adopted for anode fabrication, and the square resistance decreases with increasing dip-coating steps (Supplementary Fig. S5). The as-prepared PEDOT:PSS film is compact (Supplementary Fig. S6) and has a surface roughness of $1.57 \mathrm{~nm}$ (Supplementary Fig. S7). Due to the appropriate capillary force in the film-forming stage, uniform and bright electroluminescence can be easily realised. The current density-voltage-luminance curves of the perovskite EL fibres are shown in Fig. 2c. The QLEF still shows a considerable luminance of $\sim 100 \mathrm{~cd} \mathrm{~m}^{-2}$ at $7 \mathrm{~V}$ after being bent dozens of times (Fig. 2c), and the corresponding current efficiency is $1.67 \mathrm{~cd} \mathrm{~A}^{-1}$ (Supplementary Fig. S8). It is well known that perovskite QDs are outstanding luminance materials for next-generation highdefinition displays due to their high defect tolerance, quantum efficiency and narrow FWHM. By applying perovskite QDs as emitting materials in the electroluminescent fibre, the green electroluminescence perovskite fibre presents a narrow FWHM of $19 \mathrm{~nm}$ with chromaticity coordinates of $(0.09,0.76)$ (Fig. $2 d)$, which is the narrowest FWHM among all the reported fibreshaped electroluminescent devices (Supplementary Table S1). As shown in the insert of Fig. 2d, the operating electroluminescent perovskite fibre shows uniform and bright green emission at $7 \mathrm{~V}$. The QLEF fibres can also be easily woven into an 'N' pattern (Supplementary Fig. S9), showing great capability for wearable displays. Red fibres with uniform electroluminescence are also achieved by using the same composite strategy and exhibit chromaticity coordinates of $(0.65,0.27)$ (Fig. 2e). The electroluminescent perovskite fibre can also be being bent to a bending radius of $4.5 \mathrm{~mm}$ during operation (Fig. 2f). In addition, the perovskite fibres can be wrapped around a pencil (Supplementary Fig. S10) and exhibit the potential to be used as decoration. The resulting fibres can also be woven into a glove and a sweater (Supplementary Figs. S11 and S12). When used as a fibre-shaped electroluminescent device, inherent merits, such as a wide viewing angle and no extra absorbing losses, also exist for the proposed perovskite, as shown in Supplementary Fig. S13.

\section{Photodetection properties of the perovskite fibres}

Apart from the fine electroluminescence quality of the perovskite fibres, their photoresponse capability was surprising. The energy band diagram of the proposed perovskite electroluminescent fibre is proposed in Fig. 3a, indicating that the fibre is capable of photodetection. As shown in Fig. 3b, a photocurrent arises from direct green laser $(515 \mathrm{~nm}, 6.83 \mathrm{~mW})$ irradiation in single detection mode at a rather small bias. The current varies significantly from dark to light conditions between $-2 \mathrm{~V}$ and $2.5 \mathrm{~V}$ biases (Fig. 3b). In the insert of Fig. 3b, the $I-V$ plot around zero bias is amplified, which shows that the QLEF 

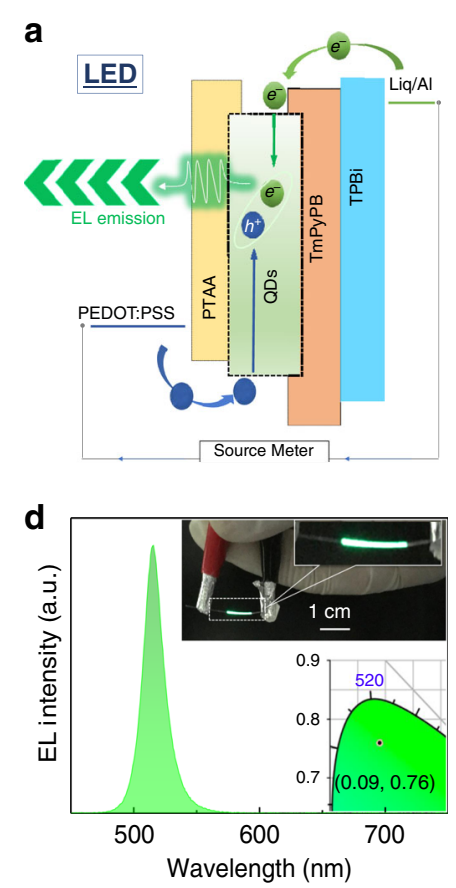

b
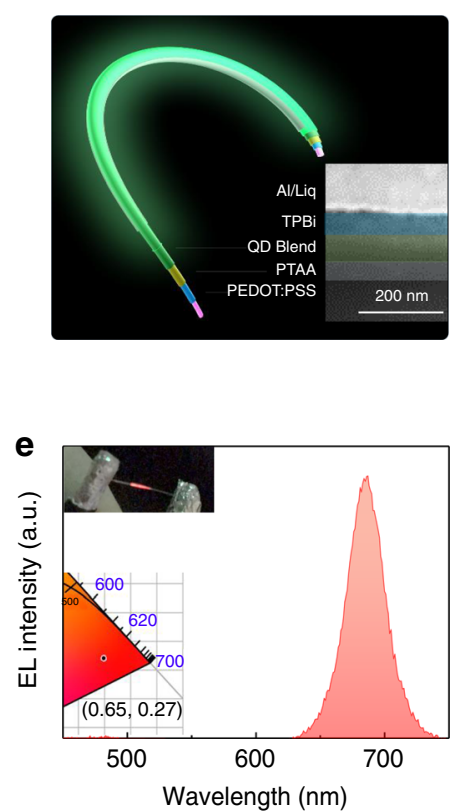

c

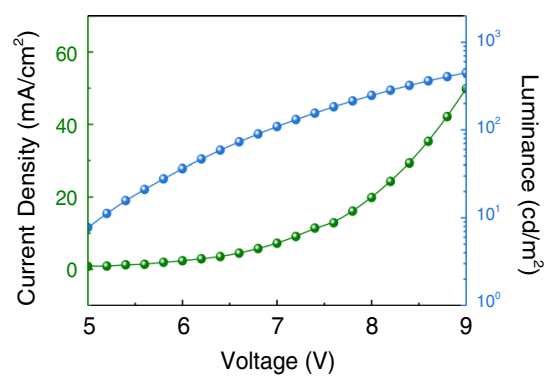

f

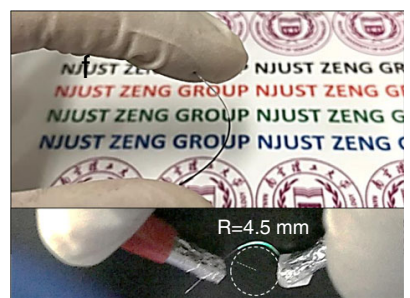

Fig. 2 Electroluminescence function of the perovskite fibres. a Schematic illustration of the working mechanism (LED mode) of the lightemitting/detecting bifunctional perovskite fibre. $\mathbf{b}$ Schematic of the structure of a perovskite EL fibre. Insert, cross-sectional SEM image of a perovskite fibre. c Current density-voltage-luminance characteristics of an electroluminescent perovskite fibre. $\mathbf{d}$, e Electroluminescence spectra of a green and a red electroluminescent perovskite fibre (inset schematics: photograph of the green and red electroluminescent perovskite fibres; chromaticity coordinates of the green and red electroluminescent perovskite fibres). $\mathbf{f}$ Photographs of a bent perovskite fibre and a bent fibre in operation.

can be self-powered, with a photocurrent response at zero bias. The self-powered property can be attributed to the band curvature in the space charge region induced by the Fermi level difference between the perovskite and the surrounding materials. The accompanying built-in field drives the charge separation process and gives rise to the photocurrent at zero bias. The $I-T$ curves of the perovskite fibre under different bias conditions are shown in Fig. 3c. The $I_{\text {light }} / I_{\text {dark }}$ ratio of the QLEF is estimated to be $\sim 1.5$ under zero bias. The responsivity of the QLEF is calculated to be $1.86 \times 10^{-3} \mathrm{~mA} \mathrm{~W}^{-1}$ at a $-2 \mathrm{~V}$ bias and $3.15 \times 10^{-3} \mathrm{~mA} \mathrm{~W}^{-1}$ at a $2.5 \mathrm{~V}$ bias under $515 \mathrm{~nm}$ illumination. The corresponding external quantum efficiency (EQE) of the QLEF in PD mode is calculated to be $0.45 \times$ $10^{-3} \%$ and $0.76 \times 10^{-3} \%$, respectively. Such a mediocre performance might result from the insufficient thickness of the active layer and could be further optimised by increasing the light capture, optimising the device structure, and improving the film quality of the active layer and contact between interfaces. In addition, the perovskite fibre has a photoresponse ability under illumination by $10 \mathrm{kHz}$ square-wave modulated LD signals at a $0 \mathrm{~V}$ bias (Supplementary Fig. S14). Interestingly, the current also varies significantly at a forward bias of approximately $2 \mathrm{~V}$, which shows potential for the construction of lightemitting/detecting bifunctional devices.

\section{Full-duplex light communication based on the EL-PD bifunctional fibres}

For a light communication system, bilateral real-time data transmission between the transmitting terminal and receiving terminal is one of the most critical issues. For wearable displays consisting of fabrics, a wide viewing angle of light-emitting fibres could satisfy the demand for multiangle data transmission. However, there are hardly any demonstrations of simultaneous data acquisition and data transmission through a single fibre. Therefore, a bifunctional fibre with both light emitting and photoresponse abilities is essential for bilateral real-time data transport and is the foundation of wearable fibre-to-fibre communication. Figure $4 \mathrm{a}$ demonstrates a wearable LiFi system based on the perovskite bifunctional fibres. Clearly, there are two transceivers in the system: the transceiver on the left represents the mobile wearable terminal (MWT) based on the perovskite bifunctional fibres; the other transceiver represents the terminal that the MWT interacts with, which consists of a commercial photodiode and a laser as a signal receiver (RX) and a transmitter (TX), respectively. A signal generator is used to generate different waveforms (first set of signals: voltage signal applied on the fibre; second set of signals: voltage signal applied on the commercial laser that induces the photoresponse of the fibre) to distinguish the 


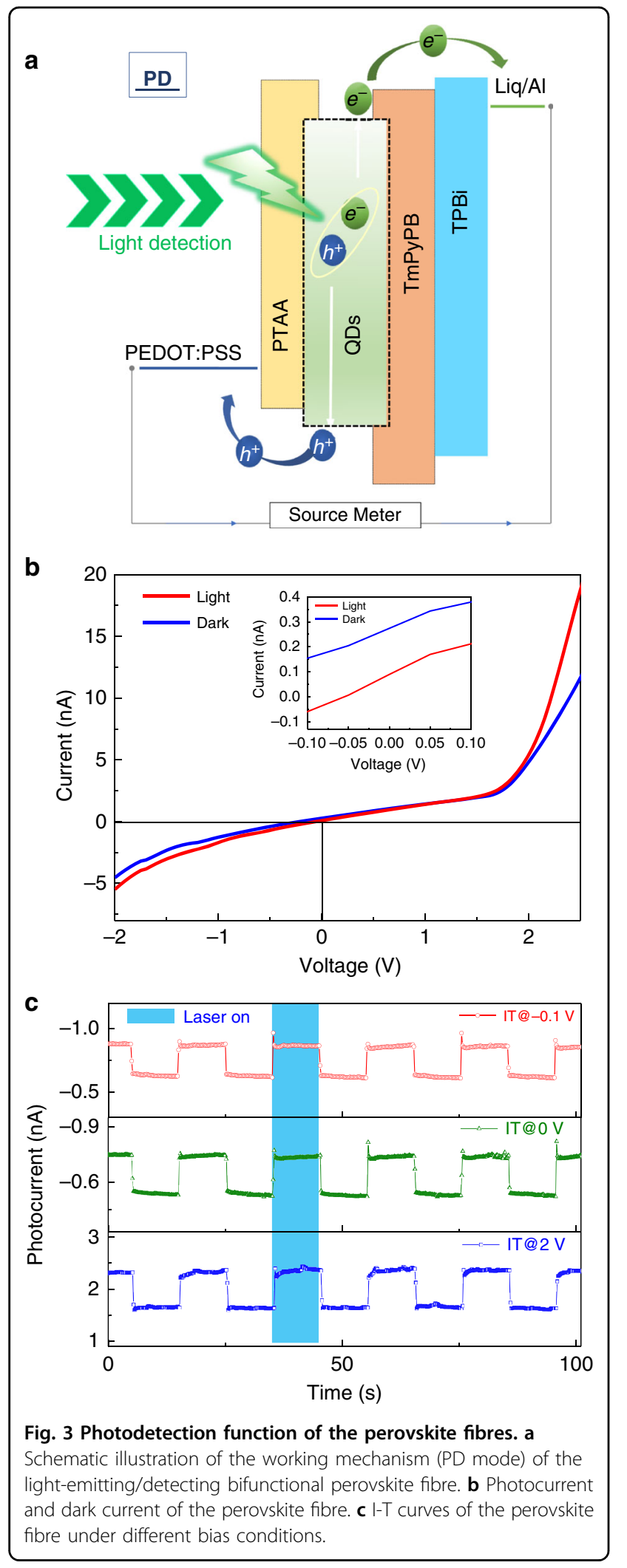

detected signal and transmitted signals of the bifunctional fibres.

Based on the above demands for a wearable LiFi system, we investigated the full-duplex LiFi properties of the asprepared bifunctional fibres. The capability for data transmission was first investigated. The perovskite fibre functions as the transmitter and is directly driven by the alternating applied voltage generated by an arbitrary waveform generator. As shown in the middle of Fig. 5a, a peak-to-peak voltage of $400 \mathrm{mV}$ and offset voltage of $7.2 \mathrm{~V}$ PRBS signal is adopted to modulate the voltage-related light-emission intensity, which contains data information. As shown at the top of Fig. 5a, the fibre shows different EL intensities and brightnesses under the modulated voltage signal. The modulated light containing data information is then received by the photodetector of the transceiver that the MWT interacts with. Afterwards, the photocurrent signals are amplified and exported to the oscilloscope (the bottom of Fig. 5a). If we assess the brighter light signals as the ' 1 ' level and less bright light signals as the ' 0 ' level, then we can clearly distinguish the 0 and 1 from the signal received by the photodetector that the transceiver MWT interacts with.

The corresponding eye diagram of the $50 \mathrm{kbit} \mathrm{s}^{-1}$ data rate clearly shows an open eye (Fig. 4c), which demonstrates the ability to transfer audio signals. An audio communication system can be easily constructed through acoustoelectric and photoelectric signal transformation with the help of external circuits (Supplementary Fig. $\mathrm{S} 15)^{23}$. However, it is difficult for the unencapsulated fibre to stick to the whole audio transfer process. Despite the mediocre data rate that one QLEF has, the integration of fibres in wearable fabric will remarkably boost the data rate, which shows that QLEFs and fabrics based on them are very suitable platforms for data transmission.

Another necessary characteristic for the bifunctional fibre used in a wearable LiFi system is the photoresponse capacity at a forward bias that can drive EL emission, which was subsequently evaluated. Figure $5 \mathrm{c}$ shows transient photoresponse signals generated by the perovskite fibre at a forward bias of $7 \mathrm{~V}$ when the QLEF was irradiated by a $405 \mathrm{~nm}$ laser pulse driven at a $10 \mathrm{kHz}$ frequency. The response and recovery processes are distinct and distinguishable. In addition, the response and recovery times are estimated to be 5 and $6 \mu$ s (defined as the time interval for the values to reach $90 \%$ and decrease to $10 \%$ of the maximum amplitude, respectively). Notably, the response time and recovery times are comparable to those of outstanding fibre-shaped perovskite photodetectors and are also sufficient for text and audio transmission in optical communication systems ${ }^{39,40}$. 


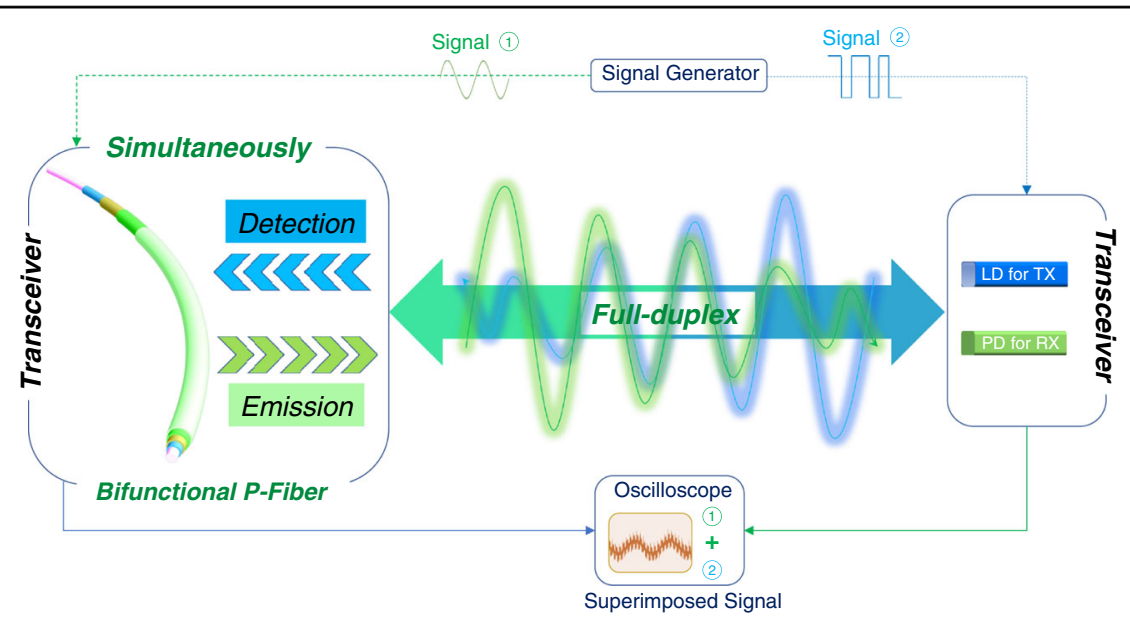

Fig. 4 Demonstration of a wearable LiFi system based on the perovskite bifunctional fibres. a Schematic illustration of the full-duplex fibrebased wearable LiFi system based on the light-emitting/detecting bifunctional perovskite fibre. The transceiver on the left represents the mobile wearable terminal (MWT) based on the perovskite bifunctional fibres; the other transceiver represents the terminal that the MWT interacts with, which consists of a commercial photodiode (PD) and a laser diode (LD) as the receiver (RX) and transmitter (TX), respectively. Two sets of signals are used to test the capability of simultaneously transmitting and receiving information. Signal 1 (sine wave) is applied on the fibre to transmit data; Signal 2 (square wave) is applied on the LD to provide the incoming data that the fibre needs to identify. A superimposed signal that contains the information of the abovementioned two sets of signals is acquired by an oscilloscope, which is clearly distinguishable.

As illustrated in Fig. 4a, we further use two sets of signals to demonstrate the simultaneously occurring data transmission and acquisition processes. First, the $405 \mathrm{~nm}$ LD driven by the square wave signal is used to irradiate the QLEF (top of Fig. 5d), and the $100 \mathrm{~Hz}$ sine wave signal is applied on the QLEF to simulate the data transmission signal (bottom of Fig. 5d). As shown in the middle of Fig. $5 \mathrm{~d}$, the superimposed signal consists of two signals: the detected signal (data acquisition) and the transmitted signal (data transmission) of the fibre-based MWT. The superimposed signals can be extracted through a selfinterference cancellation method. The simple binary data ' 1 ' and ' 0 ' are distinct, and data segments are well restored in the superimposed signal. The successful demonstration of bifunctional fibres verified the feasibility of a wearable full-duplex LiFi system based on EL-PD bifunctional perovskite fibres. Furthermore, both the electroluminescence and photodetection performance should be improved to realize bilateral real-time data transport between two fibres that belong to different interoperable LiFi terminals. In addition, different functions, such as biosensing, force sensing or other functions that are integrated into a single fibre or textiles, are also promising for future smart wearables.

In summary, we demonstrate wearable and colour tuneable perovskite light-emitting/detecting bifunctional fibres based on a hybrid strategy. The hybrid perovskite ink endows fibres with super-smooth QD films. This, combined with the small exciton binding energy and high carrier mobility of the perovskite QDs, enables the successful integration of electroluminescence and photodetection into a single fibre. The bifunctional fibres possess the narrowest luminescence spectrum of $\sim 19 \mathrm{~nm}$ and, more importantly, the capability for simultaneously transmitting and receiving information. The successful fabrication of narrow emission full-duplex LiFi fibres paves the way for the fabrication and integration of low crosstalk interoperable smart wearables.

\section{Materials and methods}

\section{Synthesis of perovskite NCs}

The synthesis of perovskite NCs follows the reported room-temperature methods ${ }^{28,41}$. In short, caesium and formamidine acetate precursors were added to $\mathrm{Pb}^{2+}$ solution, followed by $2 \mathrm{~min}$ of stirring at room temperature in air. DDAB solution was added and reacted for $3 \mathrm{~min}$, and $\mathrm{ZnBr}_{2}$ and $\mathrm{TOAB}$ were added to the crude solution and reacted for $5 \mathrm{~min}$ under continuous stirring. Ethyl acetate was used for QD purification. The precipitate was collected and redispersed in toluene, followed by the addition of poly(bis(4-phenyl)(2,4,6-trimethylphenyl) amine) (PTAA) and 1,3,5-tri[(3-pyridyl)-phen-3-yl] benzene (TmPyPB) (Xi'an Polymer Light Technology Corp, PLT).

\section{Device fabrication}

PET fibres were sequentially cleaned with acetone and distilled water by ultrasonication. A PEDOT:PSS solution (Clevios PH 1000) was mixed with 5 wt \% DMSO to improve the conductivity. Precleaned PET fibres were dipcoated in the PEDOT:PSS solution 9 times at a withdrawal speed of $60 \mathrm{~mm} \mathrm{~min}^{-1}$ to obtain the best 
a

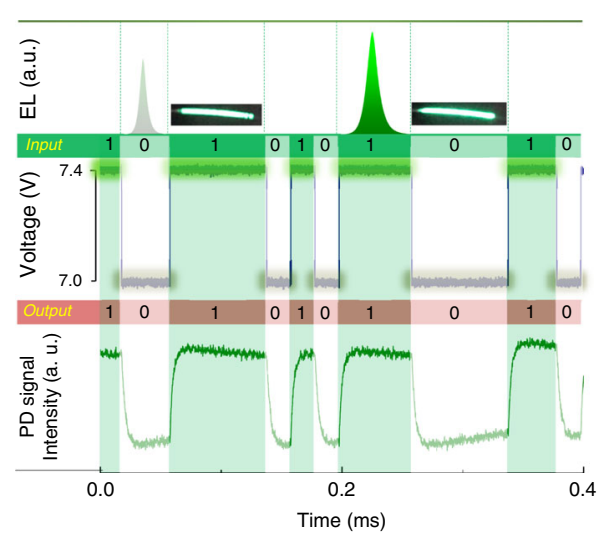

b

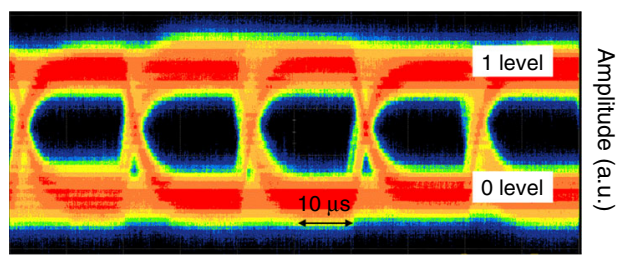

c

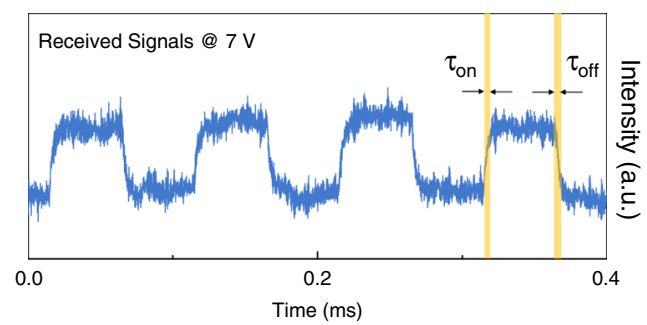

d

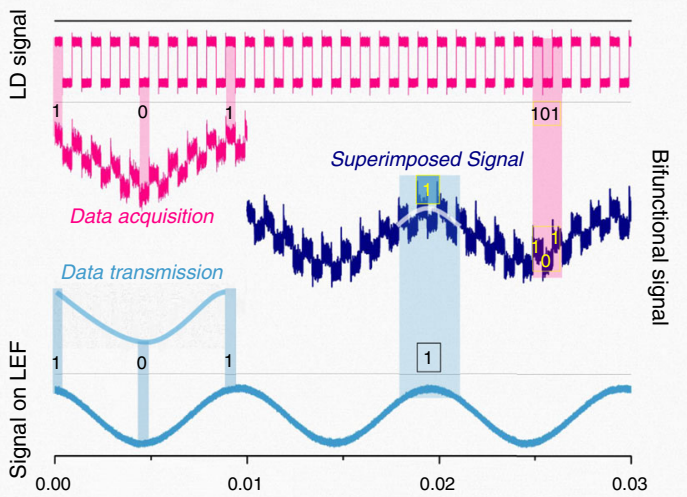

Fig. 5 Full-duplex characteristics of the bifunctional fibre LiFi. a Illustration of the light intensity-related data transmission process using the perovskite fibre as the light source. Top, EL spectrum and images of the modulated EL fibre under $7 \mathrm{~V}$ and $7.4 \mathrm{~V}$; middle, voltage signals applied on the fibre; and bottom, modulated fibre luminous signals acquired by a photodiode module. $\mathbf{b}$ Eye diagram versus data rate at $50 \mathrm{~kb} \mathrm{~s}^{-1}$ showing a clear open eye. c Square-wave signals received from the perovskite fibre under illumination by a $10 \mathrm{kHz} L D$. d Superimposed signals of the light-emitting/detecting bifunctional perovskite fibre when operating as a PD and an LED simultaneously. conductivity and then baked for $30 \mathrm{~min}$ at $110^{\circ} \mathrm{C}$. A chlorobenzene solution consisting of PTAA was dipcoated on the fibres at a withdrawal speed of $120 \mathrm{~mm}$ $\mathrm{min}^{-1}$ and then baked for $20 \mathrm{~min}$ at $120^{\circ} \mathrm{C}$. Perovskite QD inks were dip-coated on the fibres at a withdrawal speed of $120 \mathrm{~mm} \mathrm{~min}^{-1} 3$ times and then baked for $15 \mathrm{~min}$ at $60^{\circ} \mathrm{C}$. All dip-coating processes were performed in open air. Liq/Al $(3 \mathrm{~nm} / 100 \mathrm{~nm})$ were deposited by thermal evaporation under a high vacuum of $2 \times 10^{-4} \mathrm{~Pa}$. The device active area was defined by the overlapping area between the PEDOT:PSS and Al electrodes.

\section{Characterization and device measurements}

Cross sections of the perovskite fibre were characterized by a Zeiss Auriga FIB-SEM system. The UV-Vis spectra of the perovskite QD solutions were measured by a Shimadzu UV-3600 UV/VIS/NIR spectrophotometer. SEM images were obtained by using a FEI Quanta 250FEG system, and AFM images were acquired by a Bruker Multimode 8 AFM system. The PL spectra of the QDs were measured by using an Agilent Cary Eclipse spectrometer. The photodetector characteristics were measured by a Keysight B1500A semiconductor analyser. The EL spectra and $L-J-V$ characteristics were collected by using a Keithley 2400 source metre, a spectroradiometer (Cs-2000, Konica Minolta), and a close-up lens at room temperature. The viscosities of the pure and moleculedoped QD solutions were acquired on a Malvern Rheometer Instrument (Kinexus Lab+, UK). The surface tensions of the pure and molecule-doped QD solutions were measured using a Kibron EZ Pi-Plus tensiometer. The light communication system consisted of an oscilloscope (DSO9254A, Keysight), a $405 \mathrm{~nm}$ OBIS laser, an arbitrary waveform generator (33622 A, KEYSIGHT) and a photodiode module (C12702-11, Hamamatsu).

\section{Acknowledgements}

This work was financially supported by NSFC (51922049, 61725402, 61604074), the National Key Research and Development Program of China (2016YFB0401701), the Natural Science Foundation of Jiangsu Province (BK20180020), the Fundamental Research Funds for the Central Universities (30917011202), PAPD of Jiangsu Higher Education Institutions, the National 'ten thousand talents plan' leading talents (No. W03020394), and the Six top talent innovation teams of Jiangsu Province (No. TD-XCL-004). The authors also thank Prof. Wenming Su (Suzhou Institute of Nano-Tech and Nano-Bionics) for his help with the viscosity and surface tension measurements and discussion.

\section{Author contributions}

Q.S.S., J.Z.S., Y.J.W. and H.B.Z. conceived and designed the experiments. T.T.W. L.M.X. and J.H.L. synthesized the QD samples. Q.S.S. fabricated the device and performed the measurements with the help of T.F., C.T.W., Y.J. and J.X.L., Q.S.S., J.W.C., B.N.H., F.J.Z. and Y.H.D. analysed the data. Q.S.S., J.Z.S., Y.S.Z. and H.B.Z. wrote the manuscript. All authors discussed the results and commented on the manuscript. J.Z.S. and H.B.Z. supervised the project. 


\section{Data availability}

All data needed to evaluate the conclusions in the paper are present in the paper and/or the Supplementary Information. Additional data related to this paper are available from the corresponding author on request.

\section{Conflict of interest}

The authors declare that they have no conflict of interest.

Supplementary information is available for this paper at https://doi.org/ 10.1038/s41377-020-00402-8.

Received: 29 April 2020 Revised: 22 August 2020 Accepted: 24 August 2020 Published online: 16 September 2020

\section{References}

1. Pathak, P. H. et al. Visible light communication, networking, and sensing: a survey, potential and challenges. IEEE Commun. Surv. Tutor. 17, 2047-2077 (2015).

2. Abouraddy, A. F. et al. Towards multimaterial multifunctional fibres that see, hear, sense and communicate. Nat. Mater. 6, 336-347 (2007).

3. Liang, J. J. et al. Elastomeric polymer light-emitting devices and displays. Nat. Photonics 7, 817-824 (2013).

4. Rein, M. et al. Diode fibres for fabric-based optical communications. Nature 560, 214-218 (2018).

5. Rogers, J. A. et al. Materials and mechanics for stretchable electronics. Science 327, 1603-1607 (2010).

6. Son, D. et al. An integrated self-healable electronic skin system fabricated via dynamic reconstruction of a nanostructured conducting network. Nat. Nanotechnol. 13, 1057-1065 (2018).

7. Son, D. et al. Multifunctional wearable devices for diagnosis and therapy of movement disorders. Nat. Nanotechnol. 9, 397-404 (2014).

8. Wen, Z. et al. Self-powered textile for wearable electronics by hybridizing fibershaped nanogenerators, solar cells, and supercapacitors. Sci. Adv. 2, e1600097 (2016).

9. Zhang, Z. T. et al. A colour-tunable, weavable fibre-shaped polymer lightemitting electrochemical cell. Nat. Photonics 9, 233-238 (2015).

10. Behabtu, N. et al. Strong, light, multifunctional fibers of carbon nanotubes with ultrahigh conductivity. Science 339, 182-186 (2013).

11. Choi, C. et al. Twistable and stretchable sandwich structured fiber for wearable sensors and supercapacitors. Nano Lett. 16, 7677-7684 (2016).

12. Gong, S. et al. A wearable and highly sensitive pressure sensor with ultrathin gold nanowires. Nat. Commun. 5, 3132 (2014).

13. Hamedi, M. et al. Towards woven logic from organic electronic fibres. Nat. Mater. 6, 357-362 (2007).

14. Hamedi, M. et al. Fiber-embedded electrolyte-gated field-effect transistors for e-textiles. Adv. Mater. 21, 573-577 (2009).

15. Kwon, S. et al. Weavable and highly efficient organic light-emitting fibers for wearable electronics: a scalable, low-temperature process. Nano Lett. 18, 347-356 (2018)

16. Lee, M. R. et al. Solar power wires based on organic photovoltaic materials. Science 324, 232-235 (2009).

17. Wang, X. F. et al. Fiber-based flexible all-solid-state asymmetric supercapacitors for integrated photodetecting system. Angew. Chem. Int. Ed. 53, 1849-1853 (2014).

18. Hu, B. et al. Textile-based flexible electroluminescent devices. Adv. Funct. Mater. 21, 305-311 (2011).
19. O'Connor, B. et al. Fiber shaped light emitting device. Adv. Mater. 19 3897-3900 (2007)

20. Pei, Q. B. et al. Polymer light-emitting electrochemical cells. Science $\mathbf{2 6 9}$, 1086-1088 (1995)

21. Oh, N. et al. Double-heterojunction nanorod light-responsive LEDs for display applications. Science 355, 616-619 (2017)

22. Bie, Y. Q. et al. A MoTe2-based light-emitting diode and photodetector for silicon photonic integrated circuits. Nat. Nanotechnol. 12, 1124 (2017).

23. Wang, Y. J. et al. Full-duplex light communication with a monolithic multicomponent system. Light.: Sci. Appl. 7, 83 (2018).

24. Zhang, M. et al. Ultrasmooth quantum dot micropatterns by a facile controllable liquid-transfer approach: low-cost fabrication of high-performance QLED. J. Am. Chem. Soc. 140, 8690-8695 (2018).

25. Dai, X. L. et al. Solution-processed, high-performance light-emitting diodes based on quantum dots. Nature 515, 96-99 (2014).

26. Li, J. H. et al. 50-Fold EQE improvement up to $6.27 \%$ of solution-processed allinorganic perovskite CsPbBr3 QLEDs via surface ligand density control. Adv. Mater. 29, 1603885 (2017)

27. Shan, Q. S. et al. High performance metal halide perovskite light-emitting diode: from material design to device optimization. Small 13, 1701770 (2017).

28. Song, J. Z. et al. Organic-inorganic hybrid passivation enables perovskite QLEDs with an EQE of 16.48\%. Adv. Mater. 30, 1805409 (2018).

29. Song, J. Z. et al. Quantum dot light-emitting diodes based on inorganic perovskite cesium lead halides ( $\left(\mathrm{CSPBX}_{3}\right)$. Adv. Mater. 27, 7162-7167 (2015).

30. Shan, Q. S. et al. All-inorganic quantum-dot light-emitting diodes based on perovskite emitters with low turn-on voltage and high humidity stability. J. Mater. Chem. C. 5, 4565-4570 (2017).

31. Sutherland, B. R. et al. Perovskite photonic sources. Nat. Photonics 10, 295-302 (2016).

32. Di, D. W. et al. Size-dependent photon emission from organometal halide perovskite nanocrystals embedded in an organic matrix. J. Phys. Chem. Lett. $\mathbf{6}$ 446-450 (2015)

33. Tong, Y. et al. Spontaneous self-assembly of perovskite nanocrystals into electronically coupled supercrystals: toward filling the green gap. Adv. Mater. 30, 1801117 (2018).

34. Manzi, A. et al. Resonantly enhanced multiple exciton generation through below-band-gap multi-photon absorption in perovskite nanocrystals. Nat. Commun. 9, 1518 (2018)

35. Brinker, C. J. et al. Fundamentals of sol-gel dip coating. Thin Solid Films 201 97-108 (1991).

36. Ko, K. J. et al. High-performance, color-tunable fiber shaped organic lightemitting diodes. Nanoscale 10, 16184-16192 (2018).

37. Kwon, S. et al. High luminance fiber-based polymer light-emitting devices by a dip-coating method. Adv. Electron. Mater. 1, 1500103 (2015).

38. Zhao, B. D. et al. High-efficiency perovskite-polymer bulk heterostructure lightemitting diodes. Nat. Photonics 12, 783-789 (2018).

39. Sun, H. X. et al. Ultrahigh-performance self-powered flexible doubletwisted fibrous broadband perovskite photodetector. Adv. Mater. 30 1706986 (2018).

40. Liu, X. Y. et al. Ultrafast, self-powered and charge-transport-layer-free photodetectors based on high-quality evaporated $\mathrm{CsPbBr}_{3}$ perovskites for applications in optical communication. J. Mater. Chem. C. 8 3337-3350 (2020).

41. Song, J. Z. et al. Room-temperature triple-ligand surface engineering synergistically boosts ink stability, recombination dynamics, and charge injection toward EQE-11.6\% perovskite OLEDs. Adv. Mater. 30, 1800764 (2018). 